\title{
Correction to: On the strong universal consistency of local averaging regression estimates
}

\section{Matthias Hansmann ${ }^{1} \cdot$ Michael Kohler ${ }^{1} \cdot$ Harro Walk $^{2}$}

Published online: 29 August 2018

(c) The Institute of Statistical Mathematics, Tokyo 2018

\section{Correction to: Ann Inst Stat Math https://doi.org/10.1007/s10463-018-0674-9}

There is a gap at the end of the proof of Theorem 1, since there the application of the conditional McDiarmid inequality yields

$$
J_{n}-\mathbf{E}\left\{J_{n} \mid X_{1}, \ldots, X_{n}\right\} \rightarrow 0 \text { a.s. }
$$

where $J_{n}=\int\left|\sum_{i=1}^{n} W_{n, i}(x) \cdot\left(Y_{i}-m\left(X_{i}\right)\right)\right| \mu(\mathrm{d} x)$, and not yet the assertion

$$
J_{n} \rightarrow 0 \text { a.s. }
$$

in the last step of the proof of Theorem 1.

This gap can be filled by adding into assumption (A3) the second condition

$$
\sum_{i=1}^{n} \int\left|W_{n, i}(x)\right|^{2} \mu(\mathrm{d} x) \rightarrow 0 \quad \text { a.s. }
$$

The original article can be found online at https://doi.org/10.1007/s10463-018-0674-9.

Michael Kohler

kohler@mathematik.tu-darmstadt.de

Matthias Hansmann

hansmann@mathematik.tu-darmstadt.de

Harro Walk

walk@mathematik.uni-stuttgart.de

1 Fachbereich Mathematik, Technische Universität Darmstadt, Schlossgartenstr. 7, 64289

Darmstadt, Germany

2 Fachbereich Mathematik, Universität Stuttgart, Pfaffenwaldring 57, 70569 Stuttgart, Germany 
Using this condition together with $|Y| \leq L$ a.s., it is easy to see that one has

$$
\mathbf{E}\left\{J_{n} \mid X_{1}, \ldots, X_{n}\right\} \rightarrow 0 \text { a.s. }
$$

which is still needed to obtain the assertion.

In order to verify (29) in the applications of Theorem 1, for kernel estimation in the context of Lemma 6 one notices that, up to some constant factor, the left-hand side of (29) is majorized by

$$
\int \frac{1}{1+\sum_{i=1}^{n} I_{S_{r_{1}}}\left(\frac{x-X_{i}}{h_{n}}\right)} \mu(\mathrm{d} x),
$$

which can be treated similarly to the verification of $(A 4)$ in Lemma 6 . The verification of (29) for partitioning estimation in the context of Lemma 9 is analogous.

\section{Details}

Last part of the proof of Theorem 1. It remains to show

$$
J_{n} \cdot I_{B_{n}} \rightarrow 0 \text { a.s. }
$$

Application of the conditional McDiarmid inequality as in the proof of Theorem 1 yields

$$
J_{n} \cdot I_{B_{n}}-\mathbf{E}\left\{J_{n} \cdot I_{B_{n}} \mid X_{1}, \ldots, X_{n}\right\} \rightarrow 0 \text { a.s. }
$$

Hence, it suffices to show

$$
\mathbf{E}\left\{J_{n} \mid X_{1}, \ldots, X_{n}\right\} \rightarrow 0 \text { a.s. }
$$

By the inequality of Jensen, the independence of the data and $|Y| \leq L$ a.s., we get

$$
\begin{aligned}
& \left(\mathbf{E}\left\{J_{n} \mid X_{1}, \ldots, X_{n}\right\}\right)^{2} \\
& \leq \mathbf{E}\left\{J_{n}^{2} \mid X_{1}, \ldots, X_{n}\right\} \\
& \leq \mathbf{E}\left\{\int\left|\sum_{i=1}^{n} W_{n, i}(x) \cdot\left(Y_{i}-m\left(X_{i}\right)\right)\right|^{2} \mu(\mathrm{d} x) \mid X_{1}, \ldots, X_{n}\right\} \\
& =\mathbf{E}\left\{\left|\sum_{i=1}^{n} W_{n, i}(X) \cdot\left(Y_{i}-m\left(X_{i}\right)\right)\right|^{2} \mid X_{1}, \ldots, X_{n}\right\} \\
& =\mathbf{E}\left\{\mathbf{E}\left\{\left|\sum_{i=1}^{n} W_{n, i}(X) \cdot\left(Y_{i}-m\left(X_{i}\right)\right)\right|^{2} \mid X, X_{1}, \ldots, X_{n}\right\} \mid X_{1}, \ldots, X_{n}\right\}
\end{aligned}
$$




$$
\begin{aligned}
& =\mathbf{E}\left\{\sum_{i=1}^{n} W_{n, i}(X)^{2} \cdot \mathbf{E}\left\{\left(Y_{i}-m\left(X_{i}\right)\right)^{2} \mid X, X_{1}, \ldots, X_{n}\right\} \mid X_{1}, \ldots, X_{n}\right\} \\
& \leq 4 L^{2} \cdot \mathbf{E}\left\{\sum_{i=1}^{n} W_{n, i}(X)^{2} \mid X_{1}, \ldots, X_{n}\right\} \\
& =4 L^{2} \cdot \sum_{i=1}^{n} \int\left|W_{n, i}(x)\right|^{2} \mu(\mathrm{d} x) .
\end{aligned}
$$

Thus, (30) follows from (29).

Proof of (29) in the context of Lemma 6. On the one hand, we have

$$
\sum_{i=1}^{n} W_{n, i}(x)^{2}=\frac{\sum_{i=1}^{n} K\left(\frac{x-X_{i}}{h_{n}}\right)^{2}}{\left(\sum_{j=1}^{n} K\left(\frac{x-X_{j}}{h_{n}}\right)\right)^{2}} \leq 1 .
$$

On the other hand, it holds

$$
\begin{aligned}
\sum_{i=1}^{n} W_{n, i}(x)^{2} & \leq c_{2} \cdot \frac{\sum_{i=1}^{n} K\left(\frac{x-X_{i}}{h_{n}}\right)}{\left(\sum_{j=1}^{n} K\left(\frac{x-X_{j}}{h_{n}}\right)\right)^{2}} \cdot I_{\left\{\sum_{j=1}^{n} K\left(\frac{x-X_{j}}{h_{n}}\right)>0\right\}} \\
& \leq c_{2} \cdot \frac{1}{\sum_{j=1}^{n} K\left(\frac{x-X_{j}}{h_{n}}\right)} .
\end{aligned}
$$

Consequently,

$$
\begin{aligned}
\sum_{i=1}^{n} W_{n, i}(x)^{2} & \leq \min \left\{1, c_{2} \cdot \frac{1}{\sum_{j=1}^{n} K\left(\frac{x-X_{j}}{h_{n}}\right)}\right\} \\
& \leq \min \left\{1, \frac{c_{2}}{c_{1}} \cdot \frac{1}{\sum_{j=1}^{n} I_{S_{r_{1}}}\left(\frac{x-X_{j}}{h_{n}}\right)}\right\} \\
& \leq \max \left\{1, \frac{c_{2}}{c_{1}}\right\} \cdot \min \left\{1, \frac{1}{\sum_{j=1}^{n} I_{S_{r_{1}}}\left(\frac{x-X_{j}}{h_{n}}\right)}\right\} \\
& \leq \max \left\{1, \frac{c_{2}}{c_{1}}\right\} \cdot \frac{2}{1+\sum_{j=1}^{n} I_{S_{r_{1}}}\left(\frac{x-X_{j}}{h_{n}}\right)} .
\end{aligned}
$$

Hence, it suffices to show

$$
W_{n}:=\int \frac{1}{1+\sum_{j=1}^{n} I_{S_{r_{1}}}\left(\frac{x-X_{j}}{h_{n}}\right)} \mu(\mathrm{d} x) \rightarrow 0 \text { a.s. }
$$


For any bounded sphere $S$ around 0 , by Lemma 2a and by assumption (9), we get

$$
\begin{aligned}
& \mathbf{E}\left\{\int_{S} \frac{1}{1+\sum_{j=1}^{n} I_{S_{r_{1}}}\left(\frac{x-X_{j}}{h_{n}}\right)} \mu(\mathrm{d} x)\right\} \\
& =\int_{S} \mathbf{E}\left\{\frac{1}{1+\sum_{j=1}^{n} I_{S_{r_{1}}}\left(\frac{x-X_{j}}{h_{n}}\right)}\right\} \mu(\mathrm{d} x) \\
& \leq \int_{S} \frac{1}{n \cdot \mu\left(x+h_{n} \cdot S_{r_{1}}\right)} \mu(\mathrm{d} x) \\
& \leq \frac{\text { const }}{n \cdot h_{n}^{d}} \rightarrow 0 \quad(n \rightarrow \infty),
\end{aligned}
$$

where the last inequality holds because of equation (5.1) in Györfi et al. (2002). Thus, it suffices to show

$$
W_{n}-\mathbf{E}\left\{W_{n}\right\} \rightarrow 0 \quad \text { a.s. }
$$

Analogously to the proof of (A4), with $X_{1}^{\prime}, X_{1}, \ldots, X_{n}$ independent and identically distributed and

$$
W_{n}^{\prime}:=\int \frac{1}{1+I_{S_{r_{1}}}\left(\frac{x-X_{1}^{\prime}}{h_{n}}\right)+\sum_{j=2}^{n} I_{S_{r_{1}}}\left(\frac{x-X_{j}}{h_{n}}\right)} \mu(\mathrm{d} x),
$$

by Lemma 4.2 in Kohler et al. (2003), one has

$$
\mathbf{E}\left\{\left|W_{n}-\mathbf{E}\left\{W_{n}\right\}\right|^{4}\right\} \leq c_{11} \cdot n^{2} \cdot \mathbf{E}\left\{\left(W_{n}-W_{n}^{\prime}\right)^{4}\right\} \quad(n \in \mathbb{N}) .
$$

Furthermore, by the second part of Lemma 5 one gets

$$
\begin{aligned}
& \mathbf{E}\left\{\left|W_{n}-W_{n}^{\prime}\right|^{4}\right\} \\
& \leq 16 \cdot \mathbf{E}\left\{\left(\int \frac{I_{S_{r_{1}}}\left(\frac{x-X_{1}}{h_{n}}\right)}{\left(1+\sum_{j=2}^{n} I_{S_{r_{1}}}\left(\frac{x-X_{j}}{h_{n}}\right)\right)^{2}} \mu(\mathrm{d} x)\right)^{4}\right\} \\
& \leq 16 \cdot \mathbf{E}\left\{\left(\int \frac{I_{S_{r_{1}}}\left(\frac{x-X_{1}}{h_{n}}\right)}{1+\sum_{j=2}^{n} I_{S_{r_{1}}}\left(\frac{x-X_{j}}{h_{n}}\right)} \mu(\mathrm{d} x)\right)^{4}\right\} \\
& \leq \frac{\text { const }}{n^{4}}
\end{aligned}
$$

From these relations, one obtains (32) by the Borel-Cantelli lemma and the Markov inequality. 
Proof of (29) in the context of Lemma 9. Analogously to above it suffices to show

$$
V_{n}:=\int \frac{1}{1+\sum_{j=1}^{n} I_{A_{\mathcal{P}_{n}}(x)}\left(X_{j}\right)} \mu(\mathrm{d} x) \rightarrow 0 \text { a.s. }
$$

For any bounded sphere $S$ around zero, by assumption (12) we get

$$
\int_{S} \frac{1}{n \cdot \mu\left(A_{\mathcal{P}_{n}}(x)\right)} \mu(\mathrm{d} x) \rightarrow 0 \quad(n \rightarrow \infty),
$$

from which by Lemma 2a we can conclude analogously to above

$$
\mathbf{E} V_{n} \rightarrow 0 \quad(n \rightarrow \infty)
$$

Hence, it suffices to show

$$
V_{n}-\mathbf{E}\left\{V_{n}\right\} \rightarrow 0 \quad \text { a.s. }
$$

which follows analogously to above from the second part of Lemma 7. 